\title{
COMPORTAMENTO DE SOLUÇÕES
}

\section{BEHAVIOR OF SOLUCTIONS}

Rafael Lima Oliveira; Fernando Pereira de Souza

Universidade Federal de Mato Grosso do Sul, CPTL/UFMS. e-mail: fermatmel@gmail.com.br

RESUMO - No presente trabalho é estudado os tipos de soluções das equações diferenciais ordinárias de segunda ordem com coeficientes constantes da forma $a y^{\prime \prime}+b y^{\prime}+c y=0$, com $a, b$ e $c$ constantes reais e realizado um estudo sobre o comportamento da solução $y(t)$ desta equação diferencial quando $t \rightarrow+\infty$.

Palavras-chave: equações diferenciais; soluções exponenciais; comportamento de soluções.

ABSTRACT - In this paper we studied the types of solutions of ordinary differential equations of second order with constant coefficients of the form $a y^{\prime \prime}+b y^{\prime}+c y=0$, with $a, b$ e $c$ real constants and conducted a study on the behavior of the solution $y(t)$ of this differential equation when $t \rightarrow+\infty$.

Keywords: differential equations; exponential solutions; behavior of solutions. 


\section{INTRODUÇÃO}

Nesse trabalho é feito uma abordagem sobre alguns tópicos da teoria de Equações Diferenciais. Para acompanhar seu desenvolvimento é necessário, como prérequisito, conceitos fundamentais de derivadas.

Uma equação diferencial é uma relação que envolve uma "função incógnita" e suas derivadas. Com uma vasta teoria, as equações diferenciais possuem vários campos de aplicações como física, química, biologia, engenharias dentre outras.

Um tipo particular dessas equações diferenciais é o caso das equações diferenciais de segunda ordem com coeficientes constantes, donde, possuem aplicações em situações de modelagem com problemas envolvendo física e movimento amortecido.

Nosso objetivo é encontrar um padrão entre as soluções das equações diferenciais de segunda ordem com coeficientes constantes de modo a observar o comportamento das soluções quando $t \rightarrow+\infty$ (considerando uma função $y(t)$, onde $t=$ tempo), tendo assim, informações sobre o que esperar acontecer com o experimento que foi modelado por uma equação desta forma.

\section{PRELIMINARES}

Definimos uma equação diferencial homogênea de segunda ordem com coeficientes constantes como $a y^{\prime \prime}+b y^{\prime}+c y=0, \quad$ onde $a, b, c \quad$ são constantes reais dadas.

Conforme BOYCE e DIPRIMA (2012), para encontrar as soluções destas equações, vamos supor soluções exponenciais da forma $y(t)=e^{r t}$, onde $r$ é um número a ser determinado. Sendo assim, segue que $y^{\prime}(t)=r e^{r t}$ e $y^{\prime \prime}(t)=r^{2} e^{r t}$. Substituindo essas expressões na equação diferencial em questão, obtêm-se $a r^{2}+b r+c=0 . \quad$ Desta maneira, associamos uma equação diferencial de segunda ordem com coeficientes constantes com uma equação do segundo grau que à chamaremos de equação característica associada a equação diferencial. O significado da mesma está no fato que, se $r$ é solução da equação característica então $y(t)=e^{r t}$ é solução da equação diferencial. Sendo assim, temos que analisar três casos para a equação característica, isto é, como a equação característica é uma equação do segundo grau com coeficientes reais, possui duas raízes reais distintas, duas reais iguais, ou duas complexas conjugadas. 


\section{Primeiro caso: Raízes reais distintas da} equação característica: Vamos supor que as raízes da equação característica são distintas e denotá-las por $r_{1}$ e $r_{2}$. Logo $y_{1}(t)=e^{r_{1} t} \mathrm{e}$ $y_{1}(t)=e^{r_{2} t}$ são duas soluções da equação diferencial $a y^{\prime \prime}+b y^{\prime}+c y=0$, ou ainda, a combinação linear das soluções $y(t)=$ $c_{1} y_{1}(t)+c_{2} y_{2}(t)=c_{1} e^{r_{1} t}+c_{2} e^{r_{2} t}$.

Podíamos verificar se a solução obtida $y(t)$ é realmente solução da equação diferencial em questão, para isso, devemos observar que a equação em questão é $a y^{\prime \prime}+b y^{\prime}+c y=$ $0(*)$ logo, como $y(t)=c_{1} e^{r_{1} t}+$ $c_{2} e^{r_{2} t}$, temos $y^{\prime}(t)=c_{1} r_{1} e^{r_{1} t}+c_{2} r_{2} e^{r_{2} t} \mathrm{e}$ $y^{\prime \prime(t)}=c_{1} r_{1}^{2} e^{r_{1} t}+c_{2} r_{2}^{2} e^{r_{2} t}$. Substituindo na equação $\left({ }^{*}\right)$ as expressões para $y(t), y^{\prime}(t)$ e $y^{\prime \prime}(t)$ temos,

$a\left(c_{1} r_{1}^{2} e^{r_{1} t}+c_{2} r_{2}^{2} e^{r_{2} t}\right)+b\left(c_{1} r_{1} e^{r_{1} t}+\right.$ $\left.c_{2} r_{2} e^{r_{2} t}\right)+c\left(c_{1} e^{r_{1} t}+c_{2} e^{r_{2} t}\right)=0$.

A conclusão segue-se colocando em evidência alguns termos específicos da relação acima e observando que $r_{1}$ e $r_{2}$ são raízes da equação característica.

Podemos exemplificar o que foi realizado acima com o seguinte exemplo: Resolver a equação diferencial

$$
y^{\prime \prime}+3 y^{\prime}+2 y=0,
$$

que cumpre as condições iniciais $y(0)=5$ e $y^{\prime}(0)=-7$. Para isso, deve-se observar que a equação característica associada a equação diferencial é dada por $r^{2}+3 r+2=0$. Depois de resolver a equação característica encontra-se as raízes $r_{1}=-1$ e $r_{2}=-2$.
Desta maneira, usando o que foi desenvolvido até aqui, pode-se concluir que a solução da equação diferencial é $y(t)=c_{1} e^{-t}+c_{2} e^{-2 t}$.

De acordo com as condições iniciais e do fato que $y^{\prime}(t)=-c_{1} e^{-t}-2 c_{2} e^{-2 t}$, temos que

$$
\begin{gathered}
y(0)=c_{1}+c_{2}=5, \\
y^{\prime(0)}=-c_{1}-2 c_{2}=-7,
\end{gathered}
$$

no que implica $c_{1}=3$ e $c_{2}=2$. Portanto a solução da equação diferencial com condições iniciam é dada por $y(t)=3 e^{-t}+2 e^{-2 t}$. De outro modo, podese ainda obervar o comportamento do gráfico da solução pela figura1 abaixo:

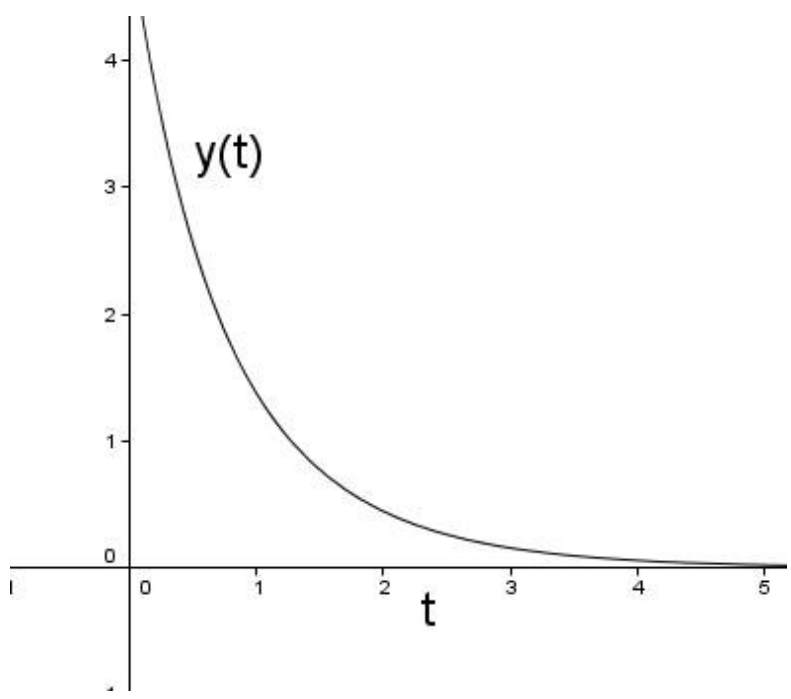

Figura1. Solução da equação $y^{\prime \prime}+3 y^{\prime}+$ $2 y=0$

\section{Segundo caso: Raízes complexas conjugadas} da equação característica: Do caso anterior, tínhamos $y(t)=c_{1} e^{r_{1} t}+c_{2} e^{r_{2} t}$. Agora, suponhamos que ao resolver a equação característica associada a equação diferencial tenha-se o discriminante $b^{2}-4 a c<0$, 
sendo

assim,

$r_{1}=\lambda+i \mu$ e $r_{2}=\lambda-i \mu$ onde $\lambda$ e $\mu$ são

números reais. Logo, as expressões correspondentes são $y_{1}(t)=e^{(\lambda+i \mu) t} \quad \mathrm{e}$ $y_{2}(t)=e^{(\lambda-i \mu) t}$. Nossa intenção neste momento, é entender as expressões $y_{1}$ e $y_{2}$. Para simplificar, usamos a fórmula $e^{i \mu}=\cos (\mu t)+i \operatorname{sen}(\mu t)$, sendo assim,

$$
\begin{aligned}
& y_{1}(t)=e^{(\lambda+i \mu) t} \\
& =e^{\lambda t}(\cos (\mu t)+i \operatorname{sen}(\mu t)) \\
& =e^{\lambda t} \cos (\mu t)+i e^{\lambda t} \operatorname{sen}(\mu t) \\
& \begin{array}{r}
y_{2}(t)=e^{(\lambda-i \mu) t}=e^{\lambda t}(\cos (\mu t)-i \operatorname{sen}(\mu t)) \\
=e^{\lambda t} \cos (\mu t)-i e^{\lambda t} \operatorname{sen}(\mu t)
\end{array}
\end{aligned}
$$

Considerando que a soma e diferença também são soluções, temos $y_{1}(t)+y_{2}(t)=2 e^{\lambda t} \cos (\mu t)$

$y_{1}(t)-y_{2}(t)=2 i e^{\lambda t} \operatorname{sen}(\mu t)$.

colocando em termos de constantes os números 2 e $2 i$, segue que a solução geral é $y(t)=c_{1} e^{\lambda t} \cos (\mu t)+c_{2} e^{\lambda t} \operatorname{sen}(\mu t)$, onde $c_{1} \mathrm{e} \quad c_{2}$ são constantes arbitrárias. Para verificar se a expressão obtida é realmente solução da equação diferencial, basta substituir conforme feito no primeiro caso.

Um exemplo para este caso pode ser visto com a equação diferencial

$$
y^{\prime \prime}+2 y^{\prime}+6 y=0,
$$

cuja equação característica associada é dada por $r^{2}+2 r+6=0$ no qual as raízes são $r_{1,2}=-1 \pm \sqrt{5} i$. Desta maneira, a solução da equação diferencial é dada por:

$$
y(t)=c_{1} e^{-t} \cos (\sqrt{5 t})+c_{2} e^{-t} \operatorname{sen}(\sqrt{5 t}) .
$$

O gráfico da solução é dado pela figura 2 a seguir:

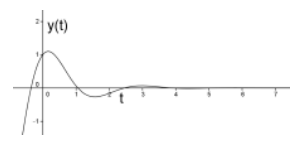

Figura2. Solução da equação $y^{\prime \prime}+2 y^{\prime}+$ $6 y=0$

\section{Terceiro Caso: Raízes repetidas da equação} característica: Este caso trata de quando as raízes $r_{1}=r_{2}$, isto é, o discriminante $b^{2}-4 a c$ da equação característica é igual a zero. Logo, vamos ter $r_{1}=r_{2}=\frac{-b}{2 a}$ e assim uma solução é $y_{1}(t)=e^{\left(\frac{-b}{2 a}\right) t}$. Vamos buscar uma segunda solução supondo $y(t)=$ $v(t) y_{1}(t)=v(t) e^{\left(\frac{-b}{2 a}\right) t}$, logo teremos:

$$
\begin{gathered}
y^{\prime}(t)=v^{\prime}(t) e^{\left(\frac{-b}{2 a}\right) t}-\frac{b}{2 a} v(t) e^{\left(\frac{-b}{2 a}\right) t}, \\
y^{\prime \prime}(t)=v^{\prime \prime(t) e^{\left(\frac{-b}{2 a}\right) t}}-\frac{b}{2 a} v^{\prime}(t) e^{\left(\frac{-b}{2 a}\right) t} \\
+\frac{b^{2}}{4 a^{2}} v(t) e^{\left(\frac{-b}{2 a}\right) t} .
\end{gathered}
$$

Substituindo na equação diferencial em questão as expressões precedentes $\left(y^{\prime}\right.$, $\left.y^{\prime \prime}\right)$ e realizando simplificações necessárias, encontra-se $v(t)=c_{1} t+c_{2}$, desta maneira, a solução segue como $y(t)=\left(c_{1} t+c_{2}\right) e^{\left(\frac{-b}{2 a}\right) t}$.

Um exemplo sobre essa situação pode ser visto como: Resolver a equação diferencial

$$
y^{\prime \prime}+4 y^{\prime}+4 y=0
$$


Para isso, deve-se observar que a equação característica associada a equação diferencial é dada por $r^{2}+4 r+4=0$. Depois de resolver a equação característica encontramse as raízes $r_{1}=r_{2}=-2$. Desta maneira, usando o que foi desenvolvido até aqui, pode-se concluir que a solução da equação diferencial

$$
y(t)=c_{1} t e^{-2 t}+c_{2} e^{-2 t} .
$$

De outro modo, pode-se ainda obervar o comportamento do gráfico da solução pela figura3 a seguir, onde vamos supor

$$
\text { constantes } \quad c_{1}=c_{2}=1 \text {. }
$$

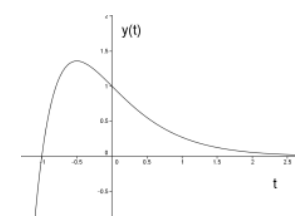

Figura3. Solução da equação $y^{\prime \prime}+4 y^{\prime}+$ $4 y=0$.

3 RESULTADOS

PRINCIPAIS: COMPORTAMENTO DAS SOLUÇÕES SEGUNDO AS RAÍZES OBTIDAS

O principal interesse agora é estudar o comportamento das soluções da equação diferencial $a y^{\prime \prime}+b y^{\prime}+c y=0$ onde vamos supor $a, b$ e $c$ constantes positivas e diferentes de zero. Para este estudo é necessário analisar três casos, como já havíamos considerado anteriormente, isto é, quando as raízes da equação característica são reais distintas, complexas conjugadas ou reais repetidas.

Raízes reais distintas: As raízes reais distintas da equação característica são dadas por

$$
r_{1}=\frac{-b+\sqrt{b^{2}-4 a c}}{2 a} \text { e } r_{2}=\frac{-b-\sqrt{b^{2}-4 a c}}{2 a} \text { e }
$$
assim as soluções da equação diferencial com coeficientes constantes são dadas pela função

$$
y(t)=c_{1} e^{r_{1} t}+c_{2} e^{r_{2} t} .
$$

De acordo com as hipóteses sobre as constantes $a, b$ e $c$ reais, temos $b>0 \Rightarrow b^{2}>0 \Rightarrow b^{2}>b^{2}-4 a c>0$ $\Rightarrow b>\sqrt{b^{2}-4 a c} \Rightarrow-b+\sqrt{b^{2}-4 a c}<0$, além

$$
\begin{aligned}
& \text { disso, } \quad \text { tem-se que } \\
& -b-\sqrt{b^{2}-4 a c}<0 .
\end{aligned}
$$

Desta maneira,

$$
\begin{gathered}
\lim _{t \rightarrow \infty} y(t)=\lim _{t \rightarrow+\infty} c_{1} e^{r_{1} t}+c_{2} e^{r_{2} t} \\
=\lim _{t \rightarrow+\infty} c_{1} e^{r_{1} t}+\lim _{t \rightarrow+\infty} c_{2} e^{r_{2} t} \\
=\lim _{t \rightarrow+\infty} c_{1} e^{\left(\frac{-b+\sqrt{b^{2}-4 a c}}{2 a}\right) t} \\
+\lim _{t \rightarrow+\infty} c_{2} e^{\left(\frac{-b-\sqrt{b^{2}-4 a c}}{2 a}\right) t} \\
=0+0=0 .
\end{gathered}
$$

O que acabamos de mostrar foi que a solução de uma equação diferencial homogênea com coeficientes constantes cujas raízes da equação característica são reais distintas tende a 0 quanto $t \rightarrow+\infty$. Graficamente ocorre o que pode-se ver no exemplo abaixo cuja equação diferencial é dada por exemplo por $y^{\prime \prime}(t)+10 y(t)+$ 
$21 y(t)=0$, cuja equação característica é $r^{2}+10 r+21=0$ que implica solução $r_{1}=-7$ e $r_{2}=-3$ de modo que a solução é dada por $y(t)=c_{1} e^{-7 t}+c_{2} e^{-3 t}$ cujo o gráfico, condiz com o que foi mostrado acima. Observe figura

4.

Figura 4. Solução da equação $y^{\prime \prime}(t)+$ $10 y(t)+21 y(t)=0$

Raízes complexas conjugadas: Neste caso a solução de $a y^{\prime \prime}+b y^{\prime}+c y=0$ onde 0 discriminante da equação característica é $b^{2}-4 a c<0$, é dada por $y(t)=c_{1} e^{\lambda t} \cos (\mu t)+c_{2} e^{\lambda t} \operatorname{sen}(\mu t)$. Como quer-se saber o comportamento das soluções quando $t \rightarrow+\infty$, fazemos:

$$
\begin{aligned}
& \lim _{t \rightarrow+\infty} y(t) \\
= & \lim _{t \rightarrow+\infty}\left(c_{1} e^{\lambda t} \cos (\mu t)+c_{2} e^{\lambda t} \operatorname{sen}(\mu t)\right) \\
= & \lim _{t \rightarrow+\infty} c_{1} e^{\lambda t} \cos (\mu t)+\lim _{t \rightarrow+\infty} c_{2} e^{\lambda t} \operatorname{sen}(\mu t) .
\end{aligned}
$$

Do fato que $\lambda=\frac{-b}{2 a}$ e $\mu=\frac{\sqrt{b^{2}-4 a c}}{2 a}$ segue que

$$
\begin{aligned}
\lim _{t \rightarrow+\infty} y(t)= & \lim _{t \rightarrow+\infty} c_{1} e^{\lambda t} \cos (\mu t) \\
& +\lim _{t \rightarrow+\infty} c_{2} e^{\lambda t} \operatorname{sen}(\mu t)
\end{aligned}
$$

$$
\begin{aligned}
& =\lim _{t \rightarrow+\infty} c_{1} e^{\left(\frac{-b}{2 a}\right) t} \cos \left(\left(\frac{\sqrt{b^{2}-4 a c}}{2 a}\right) t\right) \\
& +\lim _{t \rightarrow+\infty} c_{2} e^{\left(\frac{-b}{2 a}\right) t} \operatorname{sen}\left(\left(\frac{\sqrt{b^{2}-4 a c}}{2 a}\right) t\right)=0,
\end{aligned}
$$

pois, tendo em vista que o cosseno é limitado e $\lim _{t \rightarrow+\infty} e^{\left(\frac{-b}{2 a}\right) t}=0$ segue que o produto também tende a zero por LIMA (2013).

Exemplificando, vamos considerar a equação diferencial $y^{\prime \prime}+2 y^{\prime}+4 y=0$. Segue que a equação característica associada é dada por $r^{2}+2 r+4=0$, cujas raízes são $r= \pm \sqrt{3} i$. Logo, conforme vimos neste texto, a solução da equação diferencial para este tipo de raiz da equação característica é dada

por

$$
y(t)=c_{1} e^{-t} \cos (\sqrt{3 t})+c_{2} e^{-t} \operatorname{sen}(\sqrt{3 t}) .
$$

Observando o gráfico da solução a seguir na figura 5 , vemos realmente que conforme $t$ é maior a solução $y(t)$ tende a 0 .

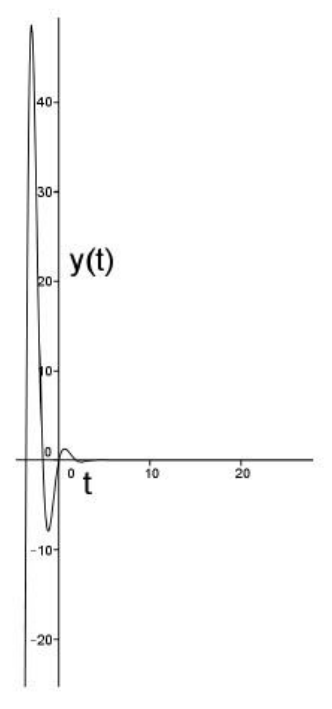

Figura 5. Solução da equação $y^{\prime \prime}+2 y^{\prime}+$ $4 y=0$. 
Raízes reais repetidas: Tem-se que a solução geral da equação diferencial $a y^{\prime \prime}+b y^{\prime}+c y=0$ com o discriminante da equação característica $b^{2}-4 a c$ é dada por $y(t)=c_{1} t e^{\left(\frac{-b}{2 a}\right) t}+c_{2} e^{\left(\frac{-b}{2 a}\right) t}, \log 0$ comportamento da solução é dado por $\lim _{t \rightarrow+\infty} y(t)=\lim _{t \rightarrow+\infty} c_{1} t e^{\left(\frac{-b}{2 a}\right) t}+$

$$
\begin{aligned}
c_{2} e^{\left(\frac{-b}{2 a}\right) t} & =\lim _{t \rightarrow+\infty} c_{1} t e^{\left(\frac{-b}{2 a}\right) t}+\lim _{t \rightarrow+\infty} c_{2} e^{\left(\frac{-b}{2 a}\right) t} \\
& =\lim _{t \rightarrow+\infty} c_{1} t \frac{1}{e^{\left(\frac{b}{2 a}\right) t}}+0=0
\end{aligned}
$$

Vamos considerar a equação diferencial $y^{\prime \prime}+2 y^{\prime}+y=0$. Para tal equação encontramos a equação característica associada $r^{2}+2 r+1=0$ cujas raízes são $r_{1}=r_{2}=-1$. Desta forma, montamos a solução da equação diferencial, que é dada por

$$
y(t)=c_{1} t e^{-t}+c_{1} t e^{-t},
$$

cujo o gráfico é dado pela figura 6 a seguir, onde tomemos $c_{1}=c_{2}=1$ :

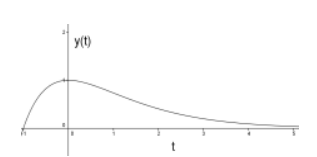

Figura6. Solução da equação $y^{\prime \prime}+2 y^{\prime}+y=$ 0 .

Repare que como mostrado acima, a solução tende a 0 conforme o valor de $t$ é maior.

\section{CONSIDERAÇÕES FINAIS}

Vamos supor uma situação de movimento amortecido chamado de problema carro-mola conforme BIANCHINI (2014) no qual vamos usar os resultados anteriores para interpretar e dar significado a essa situação problema.

Considere um carro de massa $m$ preso a uma parece por uma mola e imerso em um fluído. Coloca-se o carro em movimento puxando-o $x_{0}$ metros de sua posição de equilíbrio e soltando-o. Pela lei de Hooke, a mola exerce uma força $F_{m}$ sobre o carro proporcional à sua distenção, com coeficiente de proporcionalidade $k$. Vamos supor que o meio viscoso oferece uma força $F_{v}$ de resistência ao movimento proporcional à sua velocidade com constante de proporcionalidade $c$. Seja $x=x(t)$ a posição do carro em um instante $t$ e $v=v(t)$ sua velocidade. Uma vez iniciado o movimento, as forças atuantes no carro, isto é $F_{m}$ e $F_{v}$, tem sinais contrários. Coloquemos um referencial conforme a figura 7 a seguir:

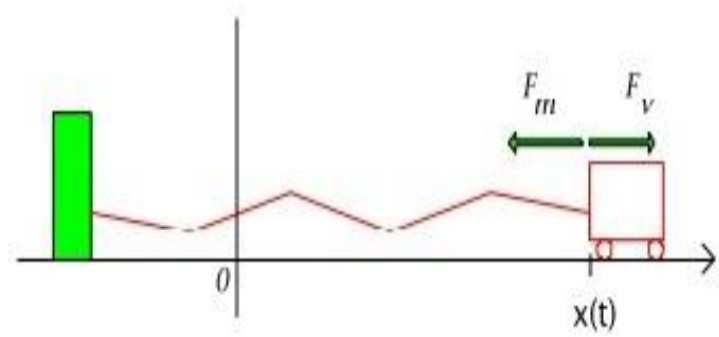

Figura7. Situação de movimento amortecido

Vamos supor que por um instante, o carro está à direita do ponto de equilíbrio. 
Neste caso, a força $F_{m}$ assume o sinal negativo e a força $F_{v}$ o sinal positivo. Acontece que, como o carro está se movimentando para a esquerda, a distância $x(t)$ da posição de equilíbrio está diminuindo, isto é, $x(t)$ está decrescendo e, portanto, sua derivada $x^{\prime}(t)$ é uma função negativa, ou seja, sua velocidade é negativa. Como $F_{v}$ é positiva, então $F_{v}=-c x^{\prime}(t)$. Logo, pela $2 a$ lei de Newton, a soma das forças atuantes no sistema carro-mola, nos dá a equação diferencial homogênea de segunda ordem com coeficientes constantes:

$$
m x^{\prime \prime}(t)+c x^{\prime}(t)+k x(t)=0 .
$$

Vamos resolver o problema considerando $m=1, c=3$ e $k=2$. Sendo assim, basta usar que a equação característica é $r^{2}+3 r+2=0$ e sendo assim, as raízes são $r_{1}=-1$ e $r_{2}=-2$. Logo, a solução geral é dada por $x(t)=c_{1} e^{-t}+c_{2} e^{-2 t}$. Neste momento, podemos interpretar o nosso resultado anterior visto que, como a equação considerada é de segunda ordem com coeficientes constantes positivos, pelo resultado anterior a solução $x(t) \rightarrow 0$ quando $t \rightarrow+\infty$, ou seja, o carro tende para sua posição de equilíbrio.

Com base nos resultados apresentados, isto é, o desenvolvimento dos três tipos de expressões para as soluções da equação diferencial de segunda ordem com coeficientes constantes, foi possível obter-se o comportamento das soluções quando a variável independente $t$ tende ao infinito, isto é, quanto um possível experimento é modelado por uma equação diferencial de segunda ordem com coeficientes constantes é possível usar este resultado de modo a contribuir com relação ao o que se esperar do experimento em longo prazo.

\section{AGRADECIMENTOS}

Ao estimado orientador, Professor doutor Fernando Pereira de Souza, pelo incansável apoio em todo o desenvolvimento do trabalho.

\section{REFERÊNCIAS}

BIANCHINI, W. Equações Diferenciais Ordinária de segunda ordem. Disponível em <www.im.ufrj.br/waldecir/calculo2/capitulo2 .pdf>. Acesso em: 09 ago. 2014.

BOYCE, W.F.; DIPRIMA, R.C. Equações diferenciais elementares e problemas de valores de contorno. 9. ed. Rio de Janeiro: LTC, 2012.

LIMA, E.L. Análise real. 12. ed. Rio de Janeiro: IMPA, 2013. 